\title{
APLICAÇÃO DE CARBOXITERAPIA NO TRATAMENTO DE FERIDAS: REVISÃO NARRATIVA
}

\author{
GONTIJO, Maria Gabriela Rodrigues ${ }^{1}$ \\ PEREIRA, Juliana Marçal Maciel de Faria ${ }^{2}$ \\ ZAPPAROLLI, Alfredo Aleixo ${ }^{2}$
}

\begin{abstract}
Recebido em: 2020.03.06
Aprovado em: 2021.04.27

ISSUE DOI: $10.3738 / 1982.2278 .3901$

RESUMO: Novas terapêuticas para as lesões de difícil cicatrização têm-se tornado necessárias e estão sendo pesquisadas. Nesse contexto, a carboxiterapia tem se destacado pelos seus efeitos sobre a microcirculação tecidual. $\mathrm{O}$ presente trabalho teve por objetivo apresentar a eficácia da carboxiterapia no tratamento de feridas e ulcerações, bem como evidenciar os cuidados para tal aplicação. Foi realizado um estudo narrativo, por meio de busca por artigos científicos encontrados nas seguintes bases de dados: Google Acadêmico, MEDLINE/Pubmed, SciELO - Scientific Electronic Library Online e LILACS/Bireme. Está pesquisa evidenciou resultados de estudos científicos que apresentam a eficácia bem como os cuidados com o emprego da técnica em feridas. Pretende-se com esse artigo auxiliar as pesquisas na área e promover a discussão sobre o tema.
\end{abstract}

Palavras chave: Pele. Tratamento. Lesões cutâneas. Cicatrização. Injeção de dióxido de Carbono.

\section{APPLICATION OF CARBOXYTHERAPY IN THE TREATMENT OF WOUNDS: NARRATIVE REVIEW}

SUMMARY: New therapies for difficult-to-heal lesions have become necessary and are being researched. In this context, carboxitherapy has stood out for its effects on tissue microcirculation. The present study aimed to present the effectiveness of carboxitherapy in the treatment of wounds and ulcerations, as well as to highlight the care for such application. A narrative study was carried out by searching for scientific articles found in the following databases: Google Scholar, MEDLINE / Pubmed, SciELO - Scientific Electronic Library Online and LILACS / Bireme. This research evidenced results of scientific studies that present the effectiveness as well as the care with the use of the technique in wounds. This article is intended to assist research in the area and promote discussion on the topic.

Keywords: Skin. Treatment. Skin lesions. Healing. Carbon dioxide injection.

\section{INTRODUÇÃO}

O dióxido de carbono é um gás liquefeito, incolor e inodoro, não inflamável levemente ácido, mais pesado que o ar e solúvel em água. Esse gás é produzido e eliminado todos os dias pelo nosso organismo através da respiração. Após sua descoberta na medicina, foram desenvolvidas novas técnicas de utilização. Seu uso terapêutico deve-se ao ano de 1932 na França. Com o método disseminado não só na França, mas a Itália e vários países europeus bem como o Brasil começaram a utilizar a técnica (KILL, 2017)

O mesmo autor citou em seu trabalho que pacientes com doenças crônicas como úlceras submetidos aos vapores de combustão da magnésia alba de forma seriada de dois a três dias

\footnotetext{
${ }^{1}$ Docente do curso de Enfermagem da Faculdade Dr. Francisco Maeda

${ }^{2}$ Discentes do curso de Medicina da Universidade de Franca
} 
sendo o primeiro método de tratamento sistêmico com $\mathrm{CO}_{2}$. Hoje em dia devido a equipamentos modernos é possível fazer o controle do fluxo injetado, tanto em tratamentos terapêuticos quanto para tratamentos estéticos.

$\mathrm{O} \mathrm{CO}_{2}$ é administrado de forma estéril, seu fluxo de pressão, velocidade e quantidade varia de acordo com cada patologia. A infusão ocorre através de uma agulha hipodérmica, por onde o gás carbônico é injetado no tecido subcutâneo. A injeção do gás provoca efeitos fisiológicos como melhora da circulação e oxigenação nos tecidos. O gás é eliminado pelo corpo depois de algum tempo, sem a necessidade de fazer repouso ou outros tipos de tratamentos complementares. Os efeitos obtidos são provenientes da ação vasomotora do $\mathrm{CO}_{2}$, que atua na microcirculação vascular do tecido conectivo. Algumas indicações da carboxiterapia que apresentaram bons resultados são: queimados, ulcerações em membros inferiores, calvície e psoríase, ou seja, doenças que se beneficiam com a melhoria da circulação (PENA, 2018).

Resultados de outros experimentos com o emprego da carboxiterapia para o tratamento de lesões crônicas contextualizam um panorama positivo quanto a aplicação dessa técnica para essa condição. Vale ressaltar que foram relatados reduzidos números de reações adversas, aumentando a expectativa de bons resultados (FERREIRA, 2019).

Neste contexto, esse estudo tem por objetivo apresentar a eficácia da carboxiterapia no tratamento de feridas e ulcerações, visando evitar a formação de cicatrizes hipertróficas, além de evidenciar os cuidados para tal aplicação, ressalta-se o pouco de estudo direcionado a esta técnica mesmo com os indícios de sua origem há um tempo bem considerável.

\section{MÉTODO}

No presente trabalho por meio de revisão bibliográfica, que tem a finalidade de reunir e sintetizar resultados de pesquisas sobre determinado tema ou questão, contribuindo para o aprofundamento do conhecimento do tema investigado.

O levantamento se fez através de busca por artigos científicos encontrados em plataformas acadêmicas reconhecidas pelo seu cunho científico como o Google Acadêmico, MEDLINE/Pubmed, SciELO - Scientific Electronic Library Online e LILACS/Bireme.

Os critérios de inclusão utilizados para a seleção da amostragem foram: textos disponibilizados na íntegra, através de acesso as bases de dados. As publicações mais condizentes foram selecionadas, incluídas por título e resumo, logo, feita uma leitura íntegra para posteriormente ser selecionado ou excluído. Foram selecionados artigos que apresentaram os seguintes descritores e/ou palavras chaves: Pele, Tratamento, Feridas, Circulação Cicatrização, Carboxiterapia. 


\section{DESENVOLVIMENTO}

\subsection{Tecido Tegumentar}

A pele atua na manutenção térmica e eletrolítica, possui essencial razão de proteção a fatores externos a partir de sua permeabilidade epidérmica e elementa-se ao sistema imunológico, nervoso e endócrino como componente constitutivo para a interação cruzada entre esses sistemas, estabelecida intracutaneamente (TRISTÃO; SALES; ROSSALES, 2018).

Além deste microbioma, a pele também possui barreiras químicas que mantém a umidade e o manto ácido da pele, barreiras físicas como queratinócitos que preservam a sua estrutura, e barreira imune compreendendo as células imunes inatas e adaptativas, que são residentes ou recrutadas para a pele. Portanto, trata-se de um órgão ativo e não apenas uma barreira mecânica inerte, apresenta diferentes níveis de proteção em uma rede funcional e altamente interconectada de células e mediadores que permitem medidas de defesa equilibradas para proteger o corpo e manter a integridade cutânea, detectando sinais de perigo, protegendo contra patógenos e montando respostas de memória (EYERICH et al.,2018).

Contudo, as lesões cutâneas continuam a exigir progresso na perspectiva de cuidado para a sua qualidade assistencial, pois afetam a população de um modo geral, onde os mais diversos princípios intrínsecos e extrínsecos oportunizam seu desenvolvimento e retardo cicatricial. Estas afecções teciduais influenciam na qualidade de vida, geram alto custo de tratamento, e por consequência, exprimem ainda significativo agravante para a saúde pública (MITTAG et al., 2017).

\subsection{LESÕES CUTÂNEAS}

As feridas representam um grande desafio à nível mundial e no cenário brasileiro, principalmente pelo ônus que acarretam e pela alta morbimortalidade desde às feridas agudas que complicam, quanto às que já são crônicas devido a algum problema associado à saúde do paciente (PEIXOTO, 2020)

Fatores classificados como extrínsecos ou intrínsecos estão relacionados com a ocorrência de feridas. Os fatores extrínsecos são representados principalmente pelos traumas externos. Já os fatores intrínsecos são predominantemente associados às comorbidades, como Diabetes Mellitus (DM), Hipertensão Arterial Sistêmica (HAS), Obesidade, Neoplasias, Vasculopatias, dentre outras (SQUIZATTO, 2017)

As feridas complexas podem ser classificadas como agudas ou crônicas. As agudas são comumente representadas por lesões que cicatrizam dentro do tempo esperado e de acordo com os estágios do processo de cicatrização. São exemplos as traumáticas e as cirúrgicas. Já as feridas crônicas são aquelas de longa duração ou que apresentam reincidência, e, normalmente, estão 
associadas às comorbidades. São exemplos as lesões diabéticas, úlceras vasculogênicas, feridas neoplásicas, dentre outras (SQUIZATTO, 2017).

Um bom exemplo das injúrias teciduais caracterizadas como crônicas, são as úlceras de membros inferiores - MMII, pois acometem pessoas de todo o mundo num percentual de 1 a $1,5 \%$ e localizam-se abaixo do joelho. Como fatores contribuintes para o surgimento dessas feridas, podem-se citar diversas causas como é o caso de agravos vasculares, neuropáticos, linfedemas, artrites reumatoides, traumas, osteomielites crônicas, anemia falciforme, vasculites, tumores cutâneos, infecções crônicas, idade superior a 69 anos dentre outras (PEIXOTO, 2020).

Em lesões cutâneas por queimadura, a extensão da perda cutânea dificulta ou mesmo impossibilita a aproximação das bordas, ocorrendo a cicatrização por segunda intenção, que é um processo mais lento, com alto risco de infecção, produzindo retração cicatricial, cicatrizes extensas e alto custo de tratamento (CRUZ, 2019).

As defesas imunológicas do corpo são muito alteradas pela queimadura. A perda da integridade cutânea é composta pela liberação de fatores inflamatórios anormais, níveis alterados de imunoglobulinas e complemento sérico, função alterada dos neutrófilos e uma redução dos linfócitos. A perda da pele também resulta em uma incapacidade de regular a temperatura corporal. Os pacientes queimados podem, portanto, exibir baixas temperaturas corporais nas primeiras horas depois da lesão, mas, à medida que o hipermetabolismo restabelece a temperatura central, o paciente queimado torna-se hipertérmico durante grande parte do período pósqueimadura, mesmo na ausência de infecção (CRUZ, 2019).

A distribuição das lesões cutâneas pode ser identificada como lesões anulares (circulares que começam em centro e se espalham em periferia), anelares (aglomeradas), isoladas, agrupadas, circinadas (torcidas, espiraladas ou serpenteadas), em íris ou em alvo (anéis concêntricos), lineares (uma linha), policíclicas (anulares que aglutinam-se) e zosteriformes (linear - herpes zoster) (SOARES, 2018).

Devido ao grande número de condições de pele que se apresentam de forma semelhante, a simples falta da identificação de algum signo na lesão pode ocasionar um diagnóstico inconcluso (LIONS; OUSLEY, 2015). Desta forma, é necessário conhecer a terminologia dos sinais morfológicos das lesões, além de ter o olhar para a coloração da pele, integridade, umidade, textura, espessura, temperatura, elasticidade, mobilidade, turgor e sensibilidade (SOARES 2018; FITZPATRICK; HIGH, 2017, LIONS; OUSLEY, 2015).

$\mathrm{Na}$ fase inflamatória, a função é o controle do sangramento e a limpeza da lesão, girando em torno de três dias. Têm início imediato com o surgimento da ferida, é totalmente dependente da atividade plaquetária da cascata de coagulação, e da liberação de alguns produtos, como substâncias vasoativas, algumas proteínas, fatores de crescimento, proteases etc. Ela se evidencia 
através dos sinais físicos de eritema, calor, edema e dor. No nível celular a inflamação é representada pela dilatação dos vasos, aumento da permeabilidade vascular, e recrutamento de leucócitos para o local da lesão (SILVA; MOREIRA 2020).

A partir do exposto, pode-se se afirmar que o reparo tissular é um processo complexo, que envolve a interação entre células que são ativadas por um grupo de mediadores de natureza diversificada, fragmentos de células e matriz extracelular, microrganismos e por alterações físicoquímicas no microambiente da lesão e das áreas a ela circunjacentes. Embora didaticamente o processo esteja divido em três fases, na realidade elas não são isoladas e sim interdependentes e sobrepostas dinamicamente no tempo (SILVA; MOREIRA 2020).

Os eventos da inflamação que levam ao desbridamento e limpeza da ferida assim, uma vez desbridada, a cicatrização das feridas entra em uma etapa conhecida como fase proliferativa de reparação. A proliferação ocorre no período de 4 a 12 dias pós-lesão. Durante este período, fibroblastos, células musculares lisas, células endoteliais e células epiteliais começam a cobrir o local da lesão. Essas células restabelecem a continuidade do tecido através da deposição da matriz, assim as células epiteliais nas margens da ferida proliferam e migram pela superfície, repondo a perda celular, formando as camadas da epiderme (MOREIRA, 2020).

\subsubsection{Maturação cutânea}

Após a epitelização, inicia a última fase do processo, a de remodelação ou maturação que é responsável pela organização do tecido cicatricial e das fibras de colágeno garantindo maior força de tensão, diminuição do tamanho da cicatriz e do eritema (LIMA 2019).

Uma ferida, em seu percurso natural, atravessa três fases, que compõem o que se conhece por processo cicatricial. Esse processo é composto por fases sequenciais e interdependentes que são as fases inflamatória, proliferativa e maturação. Por variados fatores, uma ferida pode estacionar em uma destas fases e não progredir para a cicatrização ou regeneração. Baseando-se nas fases de reparação é possível afirmar que 46,7\% das lesões aqui estudadas encontram-se estacionadas na fase proliferativa, $40 \%$ na fase inflamatória e apenas 13,3\% na fase de maturação (COIMBRA 2012; CAMPOS 2016; RIBEIRO 2019).

As lesões aqui estudadas tinham, em 63,3\% dos casos, evidência clínica ou laboratorial de infecção ou colonização. Na literatura da temática, embora não fossem mencionados dados quantitativos sobre a frequência de infecção e colonização em feridas crônicas, a infecção e a colonização são mencionadas como um importante fator que colabora com a estagnação do processo cicatricial (COIMBRA 2012; BRITO 2013; STAMBASSI 2015; PASSADOURO 2016; BARROS 2016; CAMPOS 2016; VIEIRA 2017; VIEIRA 2018 AZEVEDO 2019; RIBEIRO 2019). 
O número de feridas em que os tecidos desvitalizados foram predominantes correspondeu a $40 \%$ do total. Entende-se por tecidos desvitalizados àqueles que impedem a cicatrização (esfacelos e necroses) e que devem sofrer remoção por meios autolíticos, químicos e instrumentais, visto que a permanência destes tecidos proporciona um processo de inflamação crônica progressiva. As substâncias e coberturas com ação farmacológica são indispensáveis para intervenção nestas situações em que há estagnação do processo de reparo tecidual. (COIMBRA 2012; STAMBASSI,2015; CAMPOS,2016; RIBEIRO 2019).

\subsection{Carboxiterapia}

A cicatriz caracteriza-se por uma lesão na camada dérmica. Após uma incisão da pele, dáse início ao processo de reparo, o que se faz à custa da proliferação do tecido conjuntivo. $\mathrm{O}$ descolamento da pele provocado pelo gás, leva a perda da integridade tecidual e a exposição do colágeno, com conseqüente ativação do processo de cicatrização. Os autores relataram ainda que a Carboxiterapia também pode ser indicada para o tratamento de cicatrizes aderentes após o transcurso do processo cicatricial. Isto se justifica pela ação mecânica do gás ao promover um "descolamento" das estruturas aderidas durante o processo de reparo tecidual, proporcionando efeitos benéficos à aderência cicatricial (CRUZ, 2019)

A ação farmacológica do anidro carbônico é vasodilatação local, causando aumento do fluxo vascular e da pressão parcial de $\mathrm{O}_{2}$, por diminuir a afinidade do oxigênio pela hemoglobina, liberando, assim, mais $\mathrm{O}_{2}$ para os tecidos. Esses efeitos causam aumento de atividades oxigêniodependentes, como a fagocitose de bactérias por polimorfonucleares e produção de fibroblastos, portanto, podem resultar em melhor cicatrização (HETTIARATCHY, DZIEWULSKI 2004, LYRA, LEÃO 2012, CRUZ 2019).

A aplicação de dióxido de carbono para regenerar a cicatriz hipertrófica foi evidenciada por meio de procedimento onde utilizou-se dióxido de carbono especialmente adequado para utilização médica, com um Certificado de Conformidade pureza de 99,99\%. Aplicações subcutâneas tanto na área da ferida como na área periférica feito uso de agulhas finas, fluxo de gás máxima utilizada de $50 \mathrm{cc} / \mathrm{min}$, e o volume com taxa de aplicação máxima de $300 \mathrm{cc}$ (centímetros cúbicos) por sessão. A frequência das aplicações foi uma sessão a cada duas semanas nas primeiras seis sessões e então uma sessão de quatro em quatro semanas totalizando 10 sessões. Nos resultados, mostraram redução da cicatriz com uma precisão de $90 \%$. O efeito de dióxido de carbono baseia-se na sua potência vasodilatador. O objetivo é restaurar a função da microcirculação SALAZAR (2013 apud BRITO 2019).

Estudo histológico com a Carboxiterapia comprovou um aumento da espessura da derme, evidenciando estímulo à neocolagenase, bem como preservação total do tecido conjuntivo, 
incluindo estruturas vasculares e nervosas, ou seja, um evidente rearranjo das fibras colágenas (BENTES 2019).

O gás carbônico age quimicamente na modulação das interleucinas pró-inflamatórias, diminuindo a ação destas e aumentando das interleucinas antiinflamatórias, o que conduz um processo cicatricial normal. Simultaneamente, em razão do efeito mecânico do gás, haverá ruptura do colágeno cicatricial, que se encontra duro, denso, aderente às camadas mais profundas, e sua posterior substituição (BENTES 2019).

\subsubsection{Contraindicações}

As contraindicações giram em torno de distúrbios relacionados aos sistemas cardíaco, respiratório e renal, como infarto agudo do miocárdio, angina, asma, rinite e falência renal (OLIVEIRA 2017). Doenças autoimunes, que atingem o tecido conjuntivo - colágeno, também fazem parte do grupo de contraindicações à carboxiterapia (OLIVEIRA 2017).

A injeção de dióxido de carbono $\left(\mathrm{CO}_{2}\right)$ pode resultar em instabilidade fisiológica a partir da manipulação exagerada do produto ou dificuldades metabólicas do próprio organismo em eliminar tal excesso. $\mathrm{Na}$ intoxicação por $\mathrm{CO}_{2}$, ou a hipercapnia, a principal ação emergencial é a interrupção na infusão do gás e a estimulação da expiração pulmonar (OLIVEIRA 2017).

Durante o uso do gás carbônico em procedimento cirúrgico (laparoscopia), uma das respostas ao uso de $\mathrm{CO}_{2}$ é a taquicardia, motivada pela diminuição da circulação sanguínea em detrimento da pressão que o gás exerce sobre as estruturas anatômicas. Contudo, também se sugere que a elevação na frequência cardíaca seja em decorrência da maior absorção de $\mathrm{CO}_{2}$, por haver maior disponibilidade e, com isso, maior necessidade de eliminação do organismo (OLIVEIRA 2017).

\section{CONSIDERAÇÕES FINAIS}

Cicatrizes hipertróficas são processos de cicatrização anormal formado por deposição excessiva de matriz extracelular, especialmente colágeno e limitada à área do trauma. Há uma necessidade da prevenção da instalação da cicatriz hipertrófica. Sendo consenso que o manejo terapêutico dessa afecção permanece complexo e desafiante, apesar dos avançados conhecimentos voltado a esta problemática.

A associação do recurso terapêutico citado acima possibilita um tratamento eficaz e a consequente redução dos impactos sociais dos pacientes com problemas estéticos e déficits funcionais decorrentes da cicatriz hipertrófica. A utilização do gás provoca um enfisema subcutâneo proporcionando um descolamento da pele, isenta de traumas vasculares e nervosos, 
porém suficiente para aumentar o fluxo sanguíneo e a concentração de oxigênio local ocorrendo rearranjo das fibras de colágeno.

Com o levantamento realizado, foi possível verificar que a carboxiterapia por meio do seu estímulo químico, elétrico e traumático promove um processo inflamatório que resulta no aparecimento de leve edema e hiperemia, aumentando a capacidade de replicação dos fibroblastos e com isso a produção de fibras de colágeno e elastina.

A carborxiterapia possui respaldo na literatura para seu uso seguro e com garantias de bons resultados, no entanto não foi encontrado estudos científicos de cicatriz hipertrófica em pós queimado com o tratamento da mesma, mas é utilizada em cicatrizes hipertróficas comuns, neste contexto, é necessário maiores estudos clínicos na área de queimados utilizando-a.

\section{REFERÊNCIAS}

AZEVEDO, Isabelle Campos. de, COSTA, Roberta Kaliny de S. , HOLANDA, Cristyanne.S.M, et al. Conhecimento de enfermeiros da estratégia saúde da família sobre avaliação e tratamento de feridas oncológicas. Rev bras cancerol.. v.60, n.2, p.119-27. Disponível em: https://rbc.inca.gov.br/revista/index.php/revista/article/ view/476

BARROS, Marcelo P.L, FERREIRA, Paulo Jorge O. , MANIVA, Samia J.C.F, et al. Caracterização de feridas crônicas de um grupo de pacientes acompanhados no domicílio. Rev Interd. 2016 .1- 11. Disponível em: https://revistainterdisciplinar.

BENTES, Andréia N. Recursos fisioterapêuticos utilizados no tratamento das cicatrizes hipertróficas em pacientes queimados. Pós-graduação em Fisioterapia Dermato-Funcional Faculdade Ávila. 2019.

BRITO, Chara Keith D., Nottingham, Iale C., VICTOR, Janaína F.,et al. Úlcera venosa: avaliação clínica, orientações e cuidados com o curativo. Rev Rene. v.14, n.3, p.470- 80, 2013. Disponível em: http://www.periodicos.ufc.br/rene/ article/view/3415 . Acesso em: 12 ago. 2019.

BRITO, Juliana .Tratamento das cicatrizes hipertróficas resultantes de queimaduras. 2019. 10f. Artigo apresentado a Ceafi Pós-Graduação Pontifíca Universidade Católica de Goiás para a obtenção do título de especialista Fisioterapia Dermatofuncional

CAMPOS, Maria Genilde das C.A; De SOUZA, Alana T.O; VASCONCELOS, Josilene de M.B; et all. Feridas complexas e estomias: aspectos preventivos e manejo clínico. João Pessoa: Ideia; 2016.

COIMBRA, Fernando Ribeiro. $O$ tratamento de úlceras venosas na atenção primária: a utilização de tecnologias para o cuidado de enfermagem. [Monografia]. Corinto: Universidade Federal de Minas Gerais; 2012.

CRUZ, Gislaine Moreira da. Carboxiterapia no tratamento da cicatriz hipertrófica em pós queimado.2019. 13f. Artigo (Especialização em Fisioterapia Dermatofuncional) Ceafi Pós-Graduação Pontifíca Universidade Católica de Goiás 
EYERICH, Stefanie et al. Cutaneous Barriers and Skin Immunity: Differentiating A Connected Network. Trends in Immunology, [s.1.], v. 39, n. 4, p.315-327, abr. 2018. Doi:

http://dx.doi.org/10.1016/j.it.2018.02.004.

FERREIRA, Brenno Sousa; SILVA, Wanderson Fernandes; de SOUZA, Juarez; MORINI, Adriana Caroprezo. Efeitos da carboxiterapia na cicatrização de feridas cutâneas. Rev. Pesqui. Fisioter. v.9, n1, p.28-36, 2019. doi: 10.17267/2238-2704rpf.v9i1.2203

FITZPATRICK, James E.; HIGH, Whitney A. Urgent Care Dermatology: Symptom-Based Diagnosis E-book. [s.i.]: Elsevier, 2017. 632 p.

HETTIARATCHY, Shehan; DZIEWULSKI, Peter. ABC of burns: pathophysiology and types of burns. BMJ. v. 328, n.7453, p.1427-9, 2004.

KILL, Deise et al. A Carboxiterapia no Tratamento do Fibroedema Geloide, Varzea Grande, 10 fev. 2017. Disponível em: www.repositoriodigital.univag.com.br. Acesso em: 11 jan. 2021.

LIMA, Gabriella Keren. Ssilva. Identificação de microrganismos para controle de infecção em feridas crônicas. Monografia. Universidade Federal de Alagoas. Maceió, 2019.

LIONS, Faye ; OUSLEY, Lisa. Dermatology for the advanced practice nurse. New York, 2015.

LYRA, Marcos Carneiro, JUNIOR, Hélio Leão, NETO, Silvio Previde, ORGAES, Flávio Augusto Flório Stillitano, GONELLA, Hamilton Aleardo. Tratamento de queimaduras de carboxiterapia em modelo experimental. Rev Bras Queimaduras. v.11, n.1, p.2-5, 2012.

MITTAG, Barbara Franco et al. Cuidados com lesão de pele: Ações da Enfermagem. Estima, [s.1.], v. 15, n. 1, p.19-25, mar. 2017. Doi: http://dx.doi.org/10.5327/z1806-3144201700010004.

OLIVEIRA, Tiago da Silva .A importância da realização de exames laboratoriais como prérequisito para realização de procedimento estético de carboxiterapia. IBMR - LAUREATE INTERNATIONAL UNIVERSITIES. Rio de Janeiro, 2017.

PASSADOURO, Rui; SOUSA, Anabela; SANTOS, Cristina; COSTA, Helena et al. Características e Prevalência em Cuidados de Saúde Primários das Feridas Crônicas. Rev SPDV. [Internet]. v.74, n.1, p.45-51, 2016. Disponível em: https://doi.org/10.29021/spdv.74.1.514 Acesso em: citado em 12 ago. 2019

PENA, Luciani.; INACIO, Rodrigo Fabrizzio; ROMANO, Luis Henrique. Carboxiterapia aplicada a psoríase associada ao ácido acetilsalicílico: estudo de caso, saúde em foco, jaguariuna. 2018, ed.10, p. 690- 701. Disponível em: https://portal.unisepe.com.br/unifia/wpcontent/uploads/sites/10001/2018/08/080_CARBOXITERAPIA-APLICADA-\%C3\%80PSOR\%C3\%8DASE.pdf. Acesso em: 22 abr 2020.

PEIXÔTO JÚNIOR, Antônio; de SOUSA, Alana Oliveira; NOGUEIRA, Matheus Figueiredo; ANDRADE, Lidiane Lima. Perfil clínico e terapêutico de pacientes internados com úlceras de membros inferiores. Revista Enfermagem Atual In Derme, v. 92, n. 30, 29 jun. 2020.

RIBEIRO, Denis Fernandes da Silva. Gestão do cuidado a usuários com feridas crônicas na Atenção Básica. Revista Enfermagem Atual In Derme, v. 90, n. 28, 23 dez. 2019. 
ROSSO, Lucas Henrique; BORN, Maraísa Carine; SANTOS, Cássia Teixeira. Processo de enfermagem como norteador para a avaliação, prevenção e tratamento de lesões cutâneas. In: TRISTÃO, Fernanda Sant'ana; PADILHA, Maria Angélica Silveira.

Prevenção e tratamento de lesões cutâneas: perpectivas para o cuidado. Porto Alegre: Moriá, 2018. Cap. 1. p. 19-41.

SALAZAR, Martha Arelano. Aplicación subcutánea de dióxido de carbono para atenuación de cicatrices. Revista ECIPerú: : Revista del Encuentro Científico Internacional. v.9, n.2, p.4245, 2013.

SILVA, Denise Rivânia Vieira dos Passos ; MOREIRA, Kellyane.F. G .Intervenção de enfermagem na avaliação e tratamento de feridas em uma estratégia de saúde da família. Jan/2020. UFPI. Disponível em: https://ares.unasus.gov.br/acervo/handle/ARES/14637 . Acesso em 15 jan. 2020.

SQUIZATTO, Regina Helena et al. Perfil dos usuários atendidos em ambulatório de cuidado com feridas. Cogitare Enferm. v.22, n.1, p. 01-09, jan/mar; 2017.

http://dx.doi.org/10.5380/ce.v22i1.48472

SOARES, Gustavo Lopes. Tecnologia semióticas em enfermagem clínica dermatológica. Trabalho de Conclusão de Curso - Universidade Federal de Santa Catarina. Florianópolis,2018

STAMBASSI, Grazielle. Processo de trabalho em enfermagem: cuidado a pessoas com feridas crônicas na atenção primária à saúde. [Dissertação] Juiz de Fora: Faculdade de Enfermagem da Universidade Federal de Juiz de Fora, 2015.

VIEIRA, Chystiany Plácido de Brito, FURTADO, Alessandra Silveira, ALMEIDA, Priscilla Carvalho Dias; LUZ et al. Prevalência e caracterização de feridas crônicas em idosos assistidos na atenção básica. Rev baiana enferm. v. 31, n.3, p. e17397. Disponível em: http://dx.doi. org/10.18471/rbe.v31i3.17397 . Acesso em 12 ago. 2019

VIEIRA, Chystiany Plácido de Brito; ARAÚJO, Telma Maria Evangelista. Prevalência e fatores associados a feridas crônicas em idosos na atenção básica. Rev Esc Enferm USP. v. 52, n.1, p. e03415, 2018. Disponível em: http:// dx.doi.org/10.1590/s1980-220x2017051303415 . Acesso em 12 ago. 2019 